\title{
Motor-Incompatibility of Facial Reactions
}

\section{The Influence of Valence and Stimulus Content on Voluntary Facial Reactions}

\author{
Hedwig Eisenbarth, ${ }^{1}$ Antje B. M. Gerdes, ${ }^{2,3}$ and Georg W. Alpers ${ }^{2,3}$ \\ ${ }^{1}$ Department of Forensic Psychiatry and Psychotherapy, University of Regensburg, Germany, \\ ${ }^{2}$ Chair of Clinical and Biological Psychology and Otto Selz Institute, University of Mannheim, \\ Germany, ${ }^{3}$ Department of Psychology, University of Würzburg, Germany
}

\begin{abstract}
Emotional cues facilitate motor responses that are associated with approach or avoidance. Previous research has shown that evaluative processing of positive and negative facial expression stimuli is also linked to motor schemata of facial muscles. To further investigate the influence of different types of emotional stimuli on facial reactions, we conducted a study with pictures of emotional facial expressions (KDEF) and scenes (IAPS). Healthy participants were asked to respond to the positive or negative facial expressions (KDEF) and scenes (IAPS) with specific facial muscles in a valence-congruent (stimulus valence matches muscle related valence) or a valence-incongruent condition (stimulus valence is contrary to muscle related valence). Additionally, they were asked to rate pictures in terms of valence and arousal. Muscular response latencies were recorded by an electromyogram. Overall, response latencies were shorter in response to facial expressions than to complex pictures of scenes. For both stimulus categories, response latencies with valence-compatible muscles were shorter compared to reactions with incompatible muscles. Moreover, correlations between picture ratings and facial muscle reactions for happy facial expressions as well as positive scenes reflect a direct relationship between perceived intensity of the subjective emotional experience and physiological responding. Results replicate and extend previous research, indicating that incompatibility effects are reliable across different stimulus types and are not limited to facial mimicry.
\end{abstract}

Keywords: facial muscles, faces, incompatibility, rating

Human facial muscles are involved in direct social interactions and they can also reflect information processing. Especially the processing of emotional relevant material has been shown to be associated with accompanying reactions like heart rate change, skin conductance reactions, and facial muscles reactions (e.g., Lang, Greenwald, Bradley, \& Hamm, 1993).

According to the theory of LeDoux (1995), emotionally relevant stimuli can be processed very quickly by direct projections from the sensory thalamus to the amygdala. Adequate reactions can therefore be initiated immediately, even before stimuli are processed more detailed in the primary visual cortex. This quick processing also allows very fast initiation of physiological reactions that reflect approach or avoidance tendencies. Dimberg showed in a series of studies that pictures of positive or negative facial expressions elicit valence-specific spontaneous facial muscle reactions in the observer. That means, watching a happy facial expression results in spontaneous reactions in zygomatic major muscle, whereas watching an angry facial expression results in spontaneous reactions in corrugator supercilii muscle (Dimberg, 1982; Mojzisch et al., 2006). These reactions even occur when participants are prevented from consciously perceiving the face pictures (Dimberg \& Thunberg, 2000). It has been questioned if these reactions are due to biologically determined affect programs and therefore resemble automatic reactions. Up to now evidence prevails stating biologically determined affect programs that are important for social interaction.

Very strong evidence is provided by a study of Schilbach and colleagues (Schilbach, Eickhoff, Mojzisch, \& Vogeley, 2008) who found that spontaneous facial reactions are not only related to motor cortex activations but also to activity of the cingulate cortex, the precuneus, the hippocampus and the dorsal midbrain, all of which are areas related to social cognition. Further evidence for a more complex circuit of mimicry comes from studies finding an influence on mimicry by fear induction (Moody, Mcintosh, Mann, \& Weisser, 2007), attitudes (Likowski, Mühlberger, Seibt, Pauli, \& Weyers, 2008) and mutual gaze interaction (Schrammel, Pannasch, Graupner, Mojzisch, \& Velichkovsky, 2009). Another refutation of simple motor reflexes is the 
finding that not only facial expressions can elicit facial muscle responses. Pictures depicting emotionally relevant scenes like pictures of snakes compared to pictures of flowers (Dimberg, Thunberg, \& Grunedal, 2002) or various emotional contents of the International Affective Picture System (Lang, Bradley, \& Cuthbert, 2005; Lang et al., 1993) as well as words (Neumann, Hess, Schulz, \& Alpers, 2005) can also cause facial muscle responses. These different kinds of emotionally relevant stimuli elicit the same valence-congruent facial reactions as stimuli depicting facial expressions.

Voluntary facial expressions, although depending on different brain regions, compared to spontaneous facial reactions (Hopf, Muller-Forell, \& Hopf, 1992; McIntosh, Reichmann-Decker, Winkielman, \& Wilbarger, 2006) seem to be related to each other: Asking participants to contract facial muscles in response to pictures of facial expressions leads to faster reactions if valence of the muscle and valence of the picture match and to delayed reactions if they do not match (Dimberg et al., 2002). Dimbergs interpretation of these results is that the requested incongruent facial reaction (reacting with a valence-incongruent muscle) is performed, although the spontaneous reaction would be a different one, but it is a delayed or rather a not facilitated reaction (Dimberg et al., 2002). If spontaneous reactivity is influencing voluntary facial reactions, voluntary reactions should be slower or of smaller amplitude in response to nonmatching stimuli, both for facial stimuli and scenes of nonmatching valence. However, the social relevance of facial stimuli should be reflected in faster reactions to facial expressions compared to complex scenes. To our knowledge, up to now, there has been no direct comparison of volitional facial reactions to facial stimuli and scenes.

Another variable that could have an effect on facial reactions is the evaluation of the stimuli by the observer. In the study of Blairy, Herrera, and Hess (1999), participants who just observed pictures and classified them into emotional categories showed no correlation between rating accuracy and mimicking reactions. This study only referred to rating accuracy in terms of emotional category though, and not to arousal or valence ratings in terms of how much the subject is emotionally involved in the facial expression. If facial reactions to emotional stimuli are emotion-dependent, intensity and velocity of the spontaneous facial reactions should be related to ratings of valence and especially to ratings of arousal for the corresponding stimuli.

Including these considerations in a volitional facial reactions paradigm using facial expressions and scenes as stimuli could answer several questions: First, if stimulus congruent facial reactions can be initiated faster than incongruent facial reactions in response to emotionally relevant scenes, similarly to facial expressions stimuli. Second, whether there is a difference in latency for stimulus material between facial expressions and scenes. Third, if valence and arousal ratings for each picture are related to the facial reactions, and thus if those facial reactions can be modulated by subjective stimulus quality.

\section{Method}

\section{Participants}

36 students $(15 \mathrm{~m}, 21 \mathrm{f})$ were recruited at the University of Würzburg. Their mean age was 21.38 years $(S D=2.85$, range: $19-35)$. The mean score on social anxiety was relatively low (SPAI; $M=3.19, S D=.88$ ) in the group. Eight participants had elevated depression scores (Beck Depression Inventory score $>10$ ), as excluding them from the analysis does not change the results, results from all 36 participants are reported. Participants received chocolate for participation and participated in a lottery for a book voucher.

\section{Stimulus Material and Apparatus}

Twenty photographs of 10 female actors (Calvo \& Lundqvist, 2008; Goeleven, De Raedt, Leyman, \& Verschuere, 2008) each with two different emotional expressions (happy and angry) were chosen from the Karolinska Directed Emotional Faces $^{1}$ (KDEF; Lundqvist, Flykt, \& Ohman, 1998) as well as 10 positive and 10 negative emotional scene pictures from the International Affective Picture System ${ }^{2}$ (IAPS; Lang et al., 2005). IAPS- and KDEF-pictures of similar valence and arousal ratings were chosen according to reports of Lang and colleagues and according to ratings from previous studies of our group. Presentation of the instructions and stimuli, as well as the recording of behavioral responses was controlled by a high-precision software (Presentation, Neurobehavioral Systems Inc., Albany, CA) running on an Intel ${ }^{\circledR}$ Celeron Processor $(500 \mathrm{MHz})$. A 19 inch monitor with a resolution of $1280 \times 1024$ was used.

\section{Questionnaires}

A socio-demographic questionnaire, a questionnaire asking for stress level, difficulties, concentration, excitement and hypothesis about the aim of the study at the end of the procedure, the Beck Depression Inventory (Beck, Ward, \& Mendelson, 1961) and the Social Phobia and Anxiety Inventory (SPAI; Turner, Beidel, Dancu, \& Stanley, 1989) were administered.

\section{Procedure}

The participants were told that their task would be to react to different pictures with speech related or speech unrelated muscle groups to investigate the interaction between facial expression and speech muscles during conversations, so that they were unaware of the hypotheses during the experiment.

1 Af01has, Af07has, Af08has, Af11has, Af13has, Af17has, Af19has, Af20has, Af01ans, Af07ans, Af08ans, Af11ans, Af13ans, Af17ans, Af19ans, Af20ans.

$25600,5623,5700,5910,7230,7270,7502,8210,8502,8531,6200,6370,6570,7380,9300,9500,9571,9600,9910$. 
Table 1. Block sequences for the main study

\begin{tabular}{lllll}
\hline & \multicolumn{1}{c}{ Block 1 } & Block 2 & \multicolumn{1}{c}{ Block 3 } & \multicolumn{1}{c}{ Block 4 } \\
\hline Condition 1 & Compatible & Incompatible & Compatible & Incompatible \\
& KDEF & KDEF & IAPS & IAPS \\
Condition 2 & Incompatible & Compatible & Incompatible & Compatible \\
& KDEF & KDEF & IAPS & IAPS \\
Condition 3 & Compatible & Incompatible & Compatible & Incompatible \\
& IAPS & IAPS & KDEF & KDEF \\
Condition 4 & Incompatible & Compatible & Incompatible & Compatible \\
& IAPS & IAPS & KDEF & KDEF \\
\hline
\end{tabular}

A written informed consent was obtained of all participants. After introducing the two different muscles and calibrating the system, four practice trials with two positive and two negative pictures were presented.

The experiment consisted of four blocks each with 10 trials of happy and 10 trials of angry faces as well as 10 positive and 10 negative scenes presented in randomized order. The duration of each trial was $4.5 \mathrm{~s}$, consisting of $2.5 \mathrm{~s}$ of picture presentation and an inter-stimulus-interval of $2 \mathrm{~s}$. The participants were instructed to react as fast as possible with zygomaticus muscle contractions to positive pictures (faces or scenes) and with corrugator muscle to negative pictures in one of the blocks (compatible condition). In another block participants were asked to react with zygomaticus muscle to negative pictures and with corrugator to positive pictures (incompatible condition). Instructions and practice trials were repeated prior to each block. The order of facial expressions and scene blocks as well as of compatible and incompatible blocks was counterbalanced, leading to 4 different procedures (see Table 1). After completing the main experiment, participants rated all pictures for valence (very positive to very negative, depicted as from +4 to -4 ) and arousal (not emotionally arousing to strongly emotionally arousing, depicted as 1-9).

All procedures and data management were in accordance with the Helsinki declaration. Approval of the local ethics committee was obtained for similar studies at our lab (e.g., Neumann et al., 2005).

\section{Physiology Recording}

Facial muscle activity was bipolarly assessed with reusable $\mathrm{Ag} / \mathrm{AgCl}$ electrodes with a contact surface diameter of $5 \mathrm{~mm}$. The electrodes were placed above the zygomaticus and the corrugator muscles following the recommendations of Fridlund and Cacioppo (1986). Electromyogram (EMG) activity was recorded with a sampling rate of $1,024 \mathrm{~Hz}$, integrated and amplified using a Varioport ${ }^{\circledR}$ amplifier and recorder system (Becker Meditec, Germany). Further integration with a time constant of $20 \mathrm{~ms}$ (Blumenthal et al., 2005) and the high $(100 \mathrm{~Hz})$ and low pass $(20 \mathrm{~Hz})$ filtering of the signal (Schulz \& Alpers, 2007) was realized offline (Matlab, The MathWorks Inc., Natick, MA).

\section{Data Reduction and Analysis}

EMG responses were defined as significant deviations from a $500 \mathrm{~ms}$ baseline before each stimulus onset. In order to determine the latency of the facial response, the footpoint of the EMG response was determined with an optimized adoption (Schulz \& Alpers, 2007) of a widely used search algorithm (Globisch, Hamm, Schneider, \& Vaitl, 1993). Response latencies are calculated as logarithmized time difference of stimulus onset and EMG response onset. When participants responded with the wrong muscle or initiated the reaction before stimulus onset, the reactions were excluded from EMG latency analysis, but were later considered when calculating error rates. Response latencies and error rates were analyzed by conducting separate ANOVAs with the within-subject factors muscle (zygomaticus vs. corrugator) and picture valence (positive vs. negative). The incompatibility score was computed as the difference of compatible and incompatible reaction latencies in response to a stimulus category, divided by the sum of compatible and incompatible reaction latencies. Thus, positive values of the incompatibility score result from longer latencies in compatible conditions, whereas negative values of the incompatibility score result from longer latencies in incompatible conditions.

Picture ratings were included in repeated measures analyses using ANOVAs with a factor valence (positive vs. negative) and a factor material (KDEF vs. IAPS). Valence ratings were transformed from the -4 to +4 scale to a 1 to 9 scale for comparison reasons. For all analyses the alpha-level was set at .05. For significant effects revealed by the ANOVAS, Bonferroni corrected follow-up $t$-tests (two-tailed) were conducted and as measures of effect size, partial eta squared $\left(\eta_{\mathrm{p}}{ }^{2}\right)$, and Cohen's $d$ is reported. All analyses were done with SPSS 16.0.1 (SPSS Inc., Chicago, IL, USA).

\section{Pilot Study}

To test setting and analyses we conducted a pilot study including 25 students ( $18 \mathrm{f}, 7 \mathrm{~m}$ ) recruited at the University of Würzburg who received course credit for participation. Their mean age was 23.6 years $(S D=4.15$, range: 19 35 ). The method of the pilot study differed from the one 
described above in stimulus selection (different IAPS pictures) and in block presentation (no randomization of $\mathrm{KDEF}$ and IAPS-blocks' order).

The data of this pilot study showed that the corrugator muscle was contracted faster in response to negative than to positive pictures, $t(23)=4.13, p<.001$, whereas the zygomaticus muscle was contracted faster in response to positive than to negative pictures, $t(24)=8.08, p<.001$. Overall, responses to positive pictures are faster than to negative pictures, $F(1,23)=9.61, p=.005, \eta_{\mathrm{p}}{ }^{2}=.30$. The ANOVA of the responses to facial expression and scene pictures reveals a significant main effect of stimulus type with faster reactions in response to KDEF pictures than in response to IAPS pictures, $F(1,24)=111.47, p<.001$, $\eta_{\mathrm{p}}{ }^{2}=.83$. However, as in this pilot study the IAPS pictures partially contained facial expressions, the material effect could be underestimated. Additionally, as all participants completed first the facial expressions conditions and secondly the emotional scenes conditions, the difference in reaction time could be an order or an exhaustion effect.

\section{Results}

\section{Valence and Arousal Ratings}

Ratings of all pictures reveal expected valence and arousal differences between stimulus categories (positive vs. negative). There is no significant difference in arousal ratings between KDEF and IAPS pictures, $F(1,35)=2.21$, $p=.15, \eta_{\mathrm{p}}{ }^{2}=.06$ and between positive and negative pictures across material, $F(1,35)=0.17, p=.68, \eta_{\mathrm{p}}{ }^{2}=.01$, but a significant interaction of material and valence, $F(1,35)=31.45, \quad p=.00, \quad \eta_{\mathrm{p}}{ }^{2}=.47$. This interaction derives from a higher arousal rating for positive KDEF pictures compared to positive IAPS-pictures, $F(1,35)=$ $7.92, p=.01, \eta_{\mathrm{p}}{ }^{2}=.19$, and from a higher arousal rating of negative IAPS-pictures compared to negative KDEFpictures, $F(1,35)=26.33, p<.001, \eta_{\mathrm{p}}{ }^{2}=.43$. Differences in valence are significant for material, $F(1,35)=16.01$, $p<.001, \eta_{\mathrm{p}}{ }^{2}=.31$ with more positive ratings for the KDEF-pictures compared to the IAPS-pictures and significant valence differences for both IAPS, $F(1,35)=832.66$, $p<.001, \quad \eta_{\mathrm{p}}{ }^{2}=.96$, and KDEF pictures, $F(1,35)=$ $803.35, p<.001, \eta_{\mathrm{p}}{ }^{2}=.96$ (see Table 2$)$.
Table 2. Ratings of KDEF and IAPS pictures for valence and arousal (means and standard deviations in parenthesis)

\begin{tabular}{|c|c|c|c|}
\hline & & \multirow{2}{*}{$\frac{\mathrm{KDEF}}{M(S D)}$} & \multirow{2}{*}{$\frac{\text { IAPS }}{M(S D)}$} \\
\hline & & & \\
\hline \multirow[t]{2}{*}{ Valence } & Positive & $7.52(.83)$ & $7.25(.85)$ \\
\hline & Negative & $2.59(.55)$ & $2.00(.63)$ \\
\hline \multirow[t]{2}{*}{ Arousal } & Positive & $6.00(1.17)$ & $5.11(1.69)$ \\
\hline & Negative & $4.67(1.66)$ & $6.22(1.47)$ \\
\hline
\end{tabular}

\section{Incompatibility}

The analysis for the amplitude of the reactions reveals no significant effect of material or valence. Therefore we subsequently report only data concerning the latencies of the start of the reaction. Testing for significant differences of incompatibility scores from zero (no difference between compatible and incompatible reactions) reveals significant differences for both positive $(t(35)=6.84 ; p<.001$; $d=1.14)$ and negative facial $(t(35)=8.89 ; p<.001$; $d=1.50)$ and positive $(t(35)=2.28 ; p=.02 ; d=0.39)$ and negative $(t(35)=5.75 ; p<.001 ; d=0.97)$ scenes stimuli (see Table 3). Comparing incompatibility scores for material and valence effects in a $2 \times 2$ ANOVA (factors: material and valence) reveals no significant difference between reactions to faces or scenes, $F(1,35)=3.79$, $p=.06, \eta_{\mathrm{p}}{ }^{2}=.10$, and between positive or negative stimuli, $F(1,35)=2.20, p=.15, \eta_{\mathrm{p}}{ }^{2}=.06$, as well as no significant interaction between valence and material, $F(1,35)=0.66$, $p=.42, \eta_{\mathrm{p}}{ }^{2}=.02$.

\section{Latency Differences for Facial Stimuli and Scenes}

The $2 \times 2$ ANOVA with the factor material (facial stimuli vs. scenes) and the factor valence (positive vs. negative) results in a significant effect of material, $F(1,35)=95.99$, $p<.001, \eta_{\mathrm{p}}{ }^{2}=.73$, pointing to shorter latencies for facial stimuli compared to scenes. There is no significant difference for positive or negative scenes as well as no significant interaction of material and valence.

Table 3. Latencies of facial muscle reactions to KDEF- and IAPS-pictures of positive and negative valence for compatible and incompatible conditions (ms)

\begin{tabular}{|c|c|c|c|c|c|}
\hline & & \multicolumn{2}{|c|}{$\mathrm{KDEF}$} & \multicolumn{2}{|c|}{ IAPS } \\
\hline & & $M$ & $S D$ & $M$ & $S D$ \\
\hline \multirow[t]{2}{*}{ Compatible } & Positive & 481.59 & 151.79 & 706.38 & 217.83 \\
\hline & Negative & 457.31 & 132.21 & 661.13 & 201.66 \\
\hline \multirow[t]{2}{*}{ Incompatible } & Positive & 635.22 & 207.81 & 804.59 & 197.07 \\
\hline & Negative & 679.81 & 214.34 & 828.52 & 200.59 \\
\hline
\end{tabular}




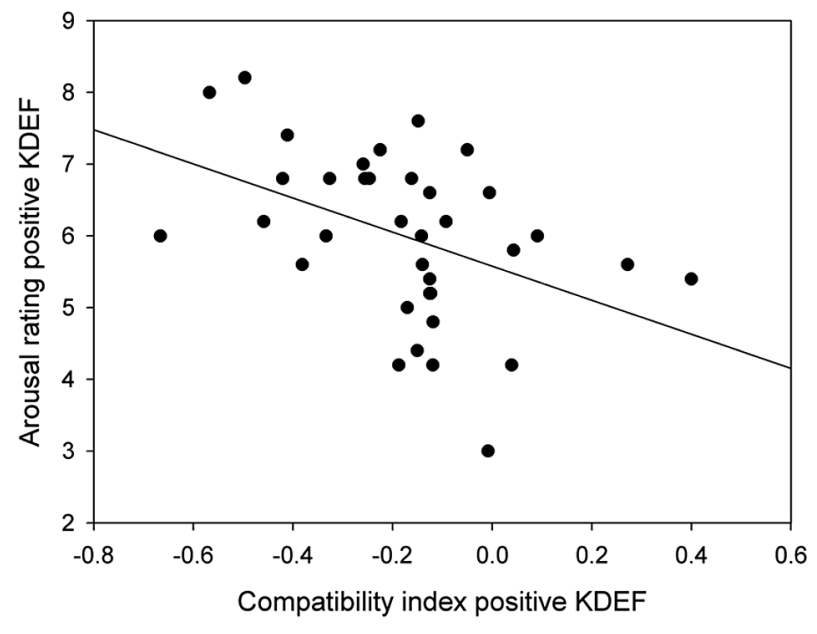

Figure 1. Correlation between arousal ratings of positive facial expressions and incompatibility indexes for latencies of reactions to positive facial expressions.

\section{Correlations Between Ratings and Reactions}

Pearson's correlation coefficients for valence and arousal ratings on the one hand and incompatibility indexes on the other reveals significant relationships between arousal ratings for positive facial expressions and the incompatibility index for reactions to positive facial expressions $(r(35)=-.44, p=.01)$ (see Figure 1). This relation is also reflected in correlations between the incompatibility index for positive facial expressions conditions and the ratings for arousal for all facial expressions' pictures $(r(35)=-.39, p=.02)$ and for arousal ratings for all positive pictures $(r(35)=-.48, p=.003)$. There is no significant correlation for negative facial expressions or for positive or negative emotional scenes.

\section{Response Errors}

Errors were defined as reactions with the incorrect muscle or a smaller reaction in the requested muscle compared to the incorrect muscle or no reaction at all and were counted for each condition. On average, participants made few errors throughout the experiment $(M=2.92, S D=5.83)$. There is no significant difference in the numbers of errors for incompatible and compatible trials, $F(1,35)=.01$, $p=.94, \eta_{\mathrm{p}}{ }^{2}=.00$. There is a significant main effect of material, $F(1,35)=8.44, p=.01, \eta_{\mathrm{p}}{ }^{2}=.19$, due to more erroneous reactions in emotional scenes' conditions compared to emotional facial expressions as well as for valence (positive vs. negative), $F(1,35)=4.93, p=.03, \eta_{\mathrm{p}}{ }^{2}=.12$, made up of more erroneous reactions to positive stimuli compared to negative stimuli.

\section{Discussion}

The aim of the present study was to directly compare volitional facial reactions in response to facial expressions and emotional scene stimuli that were valence compatible or valence incompatible. Additionally we wanted to explore the relationship between picture ratings of valence and arousal with these facial reactions in order to test the hypothesis that more intense pictures elicit faster and stronger reactions.

The incompatibility effect, that is, faster reactions to valence compatible stimuli compared to valence incompatible stimuli has been replicated for facial expressions and for emotional scenes. Thus, muscular responses involved in "smiling" are impaired while processing negative pictures and responses involved in "frowning" are impaired while processing positive ones. Furthermore, it has been shown that reactions to facial expressions can be elicited faster compared to reactions to emotional scenes, and that reactions to negative stimuli can be elicited faster compared to reactions to positive stimuli. But these differences are no more relevant in comparison of incompatible and compatible reactions. The arousal ratings for positive emotional facial expressions were correlated with the incompatibility score, which is the extent to which compatible reactions can be started faster as incompatible reactions. However, for negative facial expressions and for emotional scenes of positive and negative valence there was no correlation with the incompatibility score.

Referring to the first question, if there is a difference in reactions to facial expressions versus complex scenes, we found the same incompatibility effect for emotional scenes as well as for emotional facial expressions. Thus, our data confirm the results of Dimberg and colleagues (2002): the incompatibility effect and the valence-specific influence on facial muscle activity are not specific for facial stimuli. These results also add evidence to the hypothesis that facial reactions are biologically determined affect programs. This is in agreement with imaging data on facial muscle reactions and their role in social interaction (Hennenlotter et al., 2009; Schilbach et al., 2008). Our data further provide the possibility for direct comparison of voluntary reactions to facial and scenes stimuli, subject of the second hypothesis.

The second question was, if there is a difference in latency of reactions to facial expressions and scenes. The faster reactions to facial expressions compared to emotional scenes were not related to different arousal levels, evoked by the slides, thus a preferential processing of facial expressions can be hypothesized. This matches results of Britton and colleagues (Britton, Taylor, Sudheimer, \& Liberzon, 2006) who found in an fMRI study comparable activations in response to emotional facial expressions and to emotional scenes for most of the areas but stronger activations in response to emotional facial expressions in superior temporal gyrus, insula, and anterior cingulate cortex. The fast detection of emotional facial expressions could be due to a more complex brain activation, which could also be the basis for a faster initiation of any reactions. A recent study that used a combination of EMG and EEG adds further evidence for this suggestion (Achaibou, Pourtois, Schwartz, \& Vuilleumier, 2008): they found differences in P1 and N170 dependent on the amplitudes of the spontaneous reactions to facial expressions. Additionally, the faster reactions to facial stimuli could be due to the high relevance of facial reactions to facial cues for social interaction (Guo, 
Mahmoodi, Robertson, \& Young, 2006). However, using the incompatibility scores, the influence of material on facial reaction disappears. Thus, incompatibility costs themselves are not influenced by material or valence, which matches previous findings in reactions to word cues (Neumann et al., 2005) as well as in a stroop task using emotional words and facial expressions with no interaction of material and incompatibility (Preston \& Stansfield, 2008), and adds evidence for no difference in material and valence or an interaction in facial incompatibility to previous findings on specific pathways regulating facial incompatibility effects (e.g., Lee, Dolan, \& Critchley, 2008).

Third, according to our data there is a relationship between arousal ratings and the incompatibility score, at least for positive facial stimuli. The more intense the ratings for positive facial expressions were, the stronger the incompatibility effect in response to positive facial expressions was. This means, more intense ratings were related to facilitated reactions of smiling to smiling expressions and to impaired reactions of frowning to these positive expressions. Although this link is in accordance with our hypothesis that there should be a linear relation between arousal and reactions, the lack of any correlation concerning the negative facial expressions and both positive and negative scenes does not sufficiently approve this hypothesis. The lack of correlation could be due to differences in arousal ratings between facial and scenes stimuli. An explanation of the results for positive facial expressions could be derived from studies with high and low empathic participants. It has been shown at least for spontaneous facial reactions that those are stronger in participants scoring higher on empathy selfreport-questionnaires (e.g., Sonnby-Borgstrom, 2002). As high empathic persons tend to rate emotional facial expressions as more arousing (Sonnby-Borgstrom \& Jonsson, 2003), the stronger incompatibility effect can be influenced by the arousal of the stimuli. However, it is still in question, why the relation of rating and reactions is only present in positive facial expressions. To further investigate this relationship between arousal and incompatibility it would be suitable to combine the paradigm with a measure of physiological arousal (as e.g., SCR) and to directly compare or integrate these measures.

Due to the small number of participants further interpretations cannot be made. Furthermore, we do not have any information on empathy capabilities of the participants, which could add useful information on the relationship between arousal ratings and incompatibility. Nevertheless, our data provide further evidence that voluntary facial reactions depend on surrounding stimuli's valence and that incompatibility effects are not dependent on the kind of material (faces or scenes), nor on the valence of the material. Future studies on facial reactions should include correlations of the reactions with valence and arousal ratings to be able to understand more about their interconnections.

\section{Acknowledgments}

The first author was funded by a grant of the KonradAdenauer-Stiftung; we thank Cornelia Kruse for data collection and Katrin Blumenauer for helpful comments on the manuscript.

\section{References}

Achaibou, A., Pourtois, G., Schwartz, S., \& Vuilleumier, P. (2008). Simultaneous recording of EEG and facial muscle reactions during spontaneous emotional mimicry. Neuropsychologia, 46, 1104-1113. doi: 10.1016/j.neuropsychologia. 2007.10.019

Beck, A. T., Ward, C., \& Mendelson, M. (1961). Beck Depression Inventory (BDI). Archives of General Psychiatry, 4, 561-571.

Blairy, S., Herrera, P., \& Hess, U. (1999). Mimicry and the judgment of emotional facial expressions. Journal of Nonverbal Behavior, 23, 5-41. doi: 10.1023/a:1021370825283

Blumenthal, T. D., Cuthbert, B. N., Filion, D. L., Hackley, S., Lipp, O. V., \& van Boxtel, A. (2005). Committee report: Guidelines for human startle eyeblink electromyographic studies. Psychophysiology, 42, 1-15. doi: 10.1111/j.14698986.2005.00271.x

Britton, J. C., Taylor, S. F., Sudheimer, K. D., \& Liberzon, I. (2006). Facial expressions and complex IAPS pictures: Common and differential networks. Neuroimage, 31, 906919. doi: 10.1016/j.neuroimage.2005.12.050

Calvo, M. G., \& Lundqvist, D. (2008). Facial expressions of emotion (KDEF): Identification under different displayduration conditions. Behavior Research Methods, 40, 109115. doi: 10.3758/BRM.40.1.109

Dimberg, U. (1982). Facial reactions to facial expressions. Psychophysiology, 19, 643-647. doi: 10.1111/j.1469-8986. 1982.tb02516.x

Dimberg, U., \& Thunberg, M. (2000). Unconscious facial reactions to emotional facial expressions. Psychological Science, 11, 86. doi: 10.1111/1467-9280.00220

Dimberg, U., Thunberg, M., \& Grunedal, S. (2002). Facial reactions to emotional stimuli: Automatically controlled emotional responses. Cognition \& Emotion, 16, 449-471. doi: 10.1080/02699930143000356

Fridlund, A. J., \& Cacioppo, J. T. (1986). Guidelines for human electromyographic research. Psychophysiology, 23, 567-589. doi: 10.1111/j.1469-8986.1986.tb00676.x

Globisch, J., Hamm, A. O., Schneider, R., \& Vaitl, D. (1993). A computer program for scoring reflex eyeblink and electrodermal responses written in PASCAL. Psychophysiology, 30, S30. doi: 10.1111/j.1469-8986.1993.tb02377.x

Goeleven, E., De Raedt, R., Leyman, L., \& Verschuere, B. (2008). The Karolinska directed emotional faces: A validation study. Cognition \& Emotion, 22, 1094-1118. doi: $10.1080 / 02699930701626582$

Guo, K., Mahmoodi, S., Robertson, R., \& Young, M. (2006). Longer fixation duration while viewing face images. Experimental Brain Research, 171, 91-98. doi: 10.1007/s00221005-0248-y

Hennenlotter, A., Dresel, C., Castrop, F., Ceballos Baumann, A. O., Wohlschläger, A. M., \& Haslinger, B. (2009). The link between facial feedback and neural activity within central cercuitries of emotion - New insights from betulinum toxininduced denervation of frown muscles. Cerebral Cortex, 19, 537-542.

Hopf, H. C., Muller-Forell, W., \& Hopf, N. J. (1992). Localization of emotional and volitional facial paresis. Neurology, 42, 1918-1923.

Lang, P. J., Bradley, M. M., \& Cuthbert, B. N. (2005). International affective picture system (IAPS): Affective ratings of pictures and instruction manual. Technical Report $A$-6. Gainesville, FL: University of Florida. 
Lang, P. J., Greenwald, M. K., Bradley, M. M., \& Hamm, A. O. (1993). Looking at pictures: Affective, facial, visceral, and behavioral reactions. Psychophysiology, 30, 261-273. doi: 10.1111/j.1469-8986.1993.tb03352.x

LeDoux, J. E. (1995). Emotion: Clues from the brain. Annual Review of Psychology, 46, 209-235. doi: 10.1146/ annurev.ps.46.020195.001233

Lee, T.-W., Dolan, R. J., \& Critchley, H. D. (2008). Controlling emotional expression: Behavioral and neural correlates of nonimitative emotional responses. Cerebral Cortex, 18, 104113. doi: $10.1093 /$ cercor/bhm035

Likowski, K. U., Mühlberger, A., Seibt, B., Pauli, P., \& Weyers, P. (2008). Modulation of facial mimicry by attitudes. Journal of Experimental Social Psychology, 44, 1065-1072. doi: 10.1016/j.jesp.2007.10.007

Lundqvist, D., Flykt, A., \& Ohman, A. (1998). Karolinska directed emotional faces (KDEF). Stockholm: Department of Neurosciences.

McIntosh, D. N., Reichmann-Decker, A., Winkielman, P., \& Wilbarger, J. L. (2006). When the social mirror breaks: Deficits in automatic, but not voluntary, mimicry of emotional facial expressions in autism. Developmental Science, 9, 295-302. doi: 10.1111/j.1467-7687.2006.00492.x

Mojzisch, A., Schilbach, L., Helmert, J. R., Pannasch, S., Velichkovsky, B. M., \& Vogeley, K. (2006). The effects of self-involvement on attention, arousal, and facial expression during social interaction with virtual others: A psychophysiological study. Social Neuroscience, 1, 184-195. doi: 10.1080/17470910600985621

Moody, E. J., McIntosh, D. N., Mann, L. J., \& Weisser, K. R. (2007). More than mere mimicry? The influence of emotion on rapid facial reactions to faces. Emotion, 7, 447-457. doi: 10.1037/1528-3542.7.2.447

Neumann, R., Hess, M., Schulz, S. M., \& Alpers, G. W. (2005). Automatic behavioural responses to valence: Evidence that facial action is facilitated by evaluative processing. Cognition and Emotion, 19, 499-513. doi: 10.1080/0269993044100 0364

Preston, S. D., \& Stansfield, R. B. (2008). I know how you feel: Task-irrelevant facial expressions are spontaneously processed at a semantic level. Cognitive, Affective, \& Behavioral Neuroscience, 8, 54-64. doi: 10.3758/cabn.8.1.54

Schilbach, L., Eickhoff, S. B., Mojzisch, A., \& Vogeley, K. (2008). What's in a smile? Neural correlates of facial embodiment during social interaction. Social Neuroscience, 3, 37-50. doi: 10.1080/17470910701563228
Schrammel, F., Pannasch, S., Graupner, S.-T., Mojzisch, A., \& Velichkovsky, B. M. (2009). Virtual friend or threat? The effects of facial expression and gaze interaction on psychophysiological responses and emotional experience. Psychophysiology, 46, 922-931. doi: 10.1111/j.1469-8986.2009. 00831.x

Schulz, S. M., \& Alpers, G. W. (2007). EMGpeakfind - A MATLAB-toolbox for scoring startle eye-blink, and other EMG data. In E. Wascher, M. Falkenstein, G. Rinkenauer, \& M. Grosjean (Eds.), Psychologie und Gehirn 2007 [Psychology and the Brain, 2007]. Abstract of the Congress held at the IFADO, Dortmund, Germany, June 7-9, 2007.

Sonnby-Borgstrom, M. (2002). Automatic mimicry reactions as related to differences in emotional empathy. Scandinavian Journal of Psychology, 43, 433-443. doi: 10.1111/14679450.00312

Sonnby-Borgstrom, M., \& Jonsson, P. (2003). Models-of-self and models-of-others as related to facial muscle reactions at different levels of cognitive control. Scandinavian Journal of Psychology, 44, 141-151. doi: 10.1111/1467-9450.00332

Turner, S. M., Beidel, D. C., Dancu, C. V., \& Stanley, M. A. (1989). An empirically derived inventory to measure social fears and anxiety: The Social Phobia and Anxiety Inventory. Psychological Assessment, 1, 35-40. doi: 10.1037//10403590.1.1.35

Accepted for publication: October 4, 2010

Hedwig Eisenbarth

University of Regensburg

Department of Forensic Psychiatry and Psychotherapy

Universitätsstrasse 84

93053 Regensburg

Germany

Tel. +49 $941941-1082$

Fax +49 941 941-1085

E-mail Hedwig.eisenbarth@medbo.de 